\title{
Embedded clusters in NGC 1808 central starburst (Research Note)
}

\section{Near-infrared imaging and spectroscopy}

\author{
E. Galliano ${ }^{1,2,3}$ and D. Alloin ${ }^{4}$ \\ 1 Observatório Nacional, Rua General José Cristino 77, 20921-400 São Cristovão, Rio de Janeiro, Brazil \\ e-mail: egallian@on.br \\ 2 Departamento de Astronomia, Universidad de Chile, Casilla 36-D, Santiago, Chile \\ European Southern Observatory, Casilla 19001, Santiago 19, Chile \\ ${ }^{4}$ Laboratoire AIM, CEA/DSM-CNRS-Université Paris Diderot, IRFU/Service d'Astrophysique, Bât.709, CEA/Saclay, \\ 91191 Gif-sur-Yvette Cedex, France
}

Received 6 March 2008 / Accepted 20 May 2008

\section{ABSTRACT}

\begin{abstract}
Context. In the course of a mid-infrared imaging campaign of close-by active galaxies, we discovered the mid-infrared counterparts of bright compact radio sources in the central star-forming region of NGC 1808.

Aims. We aim at confirming that these sources are deeply embedded, young star clusters and at deriving some of their intrinsic properties.

Methods. To complement the mid-infrared images, we have collected a set of near-infrared data with ISAAC at the VLT: $J, K$ s, and $L^{\prime}$ images, as well as low-resolution, long-slit spectra for three of the sources.

Results. Surprisingly, the new images unveil a near-infrared counterpart for only one of the mid-infrared/radio sources, namely M 8 in the $L^{\prime}$ band. All the other sources are so deeply embedded that their emission does not pop out above the extended diffuse near-infrared emission. The near-infrared spectra of the sources look alike, with intense, ionised hydrogen lines. This supports the interpretation of these sources in terms of embedded young clusters. We derive extinctions and ionising photon production rates for two of the clusters.
\end{abstract}

Key words. ISM: dust, extinction - ISM: HII regions - galaxies: star clusters - galaxies: individual: NGC 1808 - infrared: galaxies

\section{Introduction}

Star clusters appear to be the most frequent mode of star formation in galaxies. Therefore, the understanding of star cluster formation and evolution is necessary for studying the stellar populations in galaxies in general. Young clusters in starburst environments have been extensively studied since the beginning of the nineties, thanks to the high angular resolution of the Hubble space telescope (HST). Yet, the very early stages of their evolution are still poorly known, as they are expected to form in dense dusty environments, hence to suffer high extinction. As it is only recently that high angular resolution instruments in the near- and mid-infrared (NIR, MIR) have become available, only a small sample of embedded young clusters is known.

In a previous study (Galliano et al. 2005) we discovered bright MIR sources within the central starburst of NGC 1808, associated with already known radio sources. We suggested that these sources were young embedded star clusters. The present research note aims at complementing the MIR dataset with new NIR data for these sources. We present NIR images of the central starburst, as well as NIR long slit spectra of three of the detected MIR/radio sources. The spectra show intense nebular emission lines and therefore bring strong support to an interpretation of the sources in terms of young embedded clusters. From the spectral measurements, some basic parameters such as extinction and ionising photon emission rate are derived for the embedded clusters.
NGC 1808 is located at a distance of 10.9 Mpc (Koribalski et al. 1996), which corresponds to a scale of 53 pc per arcsec. Its redshift is 0.003319 (Koribalski et al. 2004). The central region (inner $750 \mathrm{pc}$ ) of this galaxy is undergoing an intense episode of star formation, discussed in e.g. Tacconi-Garman et al. (1996, 2005).

\section{Observations}

With ISAAC at VLT/UT1, we collected NIR images and spectra of the circumnuclear MIR sources discovered in NGC 1808 (Galliano et al. 2005). We adopt hereafter the source nomenclature as in Galliano et al. (2005). We recorded three images: $J(1.2 \mu \mathrm{m})^{1}, K \mathrm{~s}(2.2 \mu \mathrm{m})$ and $L^{\prime}(3.8 \mu \mathrm{m})^{2}$. The image angular resolution is around $0.6^{\prime \prime}$. We also obtained low resolution long slit spectra for two slit positions. For one slit position (passing through sources $M 6$ and M 8), we obtained a $2 \mu \mathrm{m}$ spectrum $(1.8-2.4 \mu \mathrm{m})$ and a $4 \mu \mathrm{m}$ spectrum $(3.1-4.1 \mu \mathrm{m})$. For the other slit position (across the nucleus M 1 and source M 3), we could only obtain a $2 \mu \mathrm{m}$ spectrum. The spectral resolutions are $R=450$ and 360 for the $2 \mu \mathrm{m}$ and $4 \mu \mathrm{m}$ spectra respectively. The slits were positioned performing offsets referenced on the nucleus M 1, according to the measurements in Galliano et al. (2005). The data reduction was performed using the ECLIPSE

\footnotetext{
1 P074.B-0166; October 29, 30, 2004.

2 P072.B-0397; December 01, 02, 2003.
} 
dedicated routines and IRAF. The spectra were extracted through slit windows of $1.4^{\prime \prime}$ along the slit. In addition to these data, we use in this research note an archive, pipe-line reduced, WFPC2 narrow filter $F 658 \mathrm{~N}(655-661 \mathrm{~nm})$ image of the starburst, as well as the TIMMI $210.4,11.9$ and $12.9 \mu \mathrm{m}$ images presented in Galliano et al. (2005).

\section{Results}

\subsection{Images}

Figure 1 shows the central starburst in NGC $1808\left(18^{\prime \prime} \times 18^{\prime \prime}\right.$, corresponding to $1 \mathrm{kpc} \times 1 \mathrm{kpc})$ at six different wavelengths from visible to radio: $0.66 \mu \mathrm{m}, 1.2 \mu \mathrm{m}, 2.2 \mu \mathrm{m}, 3.8 \mu \mathrm{m}$, $12.9 \mu \mathrm{m}$ and $3.6 \mathrm{~cm}$. The bulk of the emission consists of an $20^{\prime \prime} \times 10^{\prime \prime}$ emitting region roughly aligned along PA $=135^{\circ}$. In the maps shown in Fig. 1 , the coordinates $\left(0^{\prime \prime} ; 0^{\prime \prime}\right)$ correspond to the brightest source $M 1$. The nature of this source has not yet been clarified in the literature. It is suggested to be a mixture of star formation and a low luminosity or fading active galactic nucleus (Krabbe et al. 2001; Jiménez-Bailón et al. 2005). This source is used for the relative registration of the different maps, assuming that the luminosity peak coincides at all studied wavelengths. The following images are displayed in Fig. 1, in order of increasing wavelength:

- the narrow filter WFPC2 images $(0.66 \mu \mathrm{m})$, which, at the redshift of NGC 1808 traces the $\mathrm{H} \alpha$ emission, and is hence a good tracer of un-embedded star formation in the region;

- the $J(1.2 \mu \mathrm{m})$ and $K \mathrm{~s}(2.2 \mu \mathrm{m})$ images which trace the photospheric emission of stars, less subject to extinction than in the visible;

- the $L^{\prime}$ band image $(3.8 \mu \mathrm{m})$ which can highlight hot dust, and hence globally traces star forming activity and AGN activity;

- the $12.9 \mu \mathrm{m}$ image which traces warm dust emission and [NeII] line emission. As for the $L^{\prime}$ band emission, the image pinpoints star forming regions and the AGN;

- the $3.6 \mathrm{~cm}$ image which corresponds to the free-free emission from HII regions, to the diffuse synchrotron emission produced by electrons escaping SN remnants and being acceleration by galactic magnetic fields, and to the likely emission from recent $\mathrm{SN}$ explosions.

The maps in Fig. 1 bring new details about the extended star forming region. Through its $\mathrm{H} \alpha$ emission (WFPC2 image), the region looks extremely patchy, likely the result of a complex distribution of star formation "nests" and of molecular/dusty clouds producing a heavy extinction. It is interesting to note the spiral arm-like structure of the F658N emission, particularly to the South of the nucleus. The $J$ and $K$ s images do not add much information to this picture, except for an enhanced contrast for some sources which look faint in the $R$ image. They do not unveil any new source, with respect to the $R$ map.

In the $L^{\prime}$ band, the region exhibits a bright diffuse emission, possibly the signature of a large amount of dust heated by the various star forming nests. Several compact sources appear on this map.

The following two images $(12.9 \mu \mathrm{m}$ and $3.6 \mathrm{~cm})$ show deeply embedded sources which do not appear on either the $R, J, K \mathrm{~s}$ or $L^{\prime}$ maps. This remark is important, showing that embedded star forming activity in starbursts can be hidden even at $4 \mu \mathrm{m}$. As already pointed out in Galliano et al. (2005), the correlation between the $12.9 \mu \mathrm{m}$ map and the $3.6 \mathrm{~cm}$ map is excellent.

Figure 2 offers zooms on two regions of interest (regions A and $\mathrm{B}$ ) both containing MIR/radio sources. In region A, M 8 is detected in $L^{\prime}$ only, while M 6 is not even detected in $L^{\prime}$. None of these sources show up in the NIR. Regarding region B, there is no detection either in $K$ s or $L^{\prime}$. Strikingly, on the $K$ s map, the location of M 3 even corresponds to a dark area in the diffuse emission.

Conversely, we note that the K knots detected by TacconiGarman et al. (1996), and which are interpreted as being embedded young stellar clusters too, are not detected on the $12.9 \mu \mathrm{m}$ map.

Hence, there are two types of embedded clusters in NGC 1808: one bright in the MIR, bright in the radio and faint in the NIR, and the other bright in the NIR and faint in the MIR and the radio. This dichotomy can simply be explained if we consider that MIR/radio clusters are at an earlier evolution stage than the sole K clusters: at a few million years of age, the clusters are still deeply embedded in their formation material, which is a mixture of ionised gas (source of the radio emission and nebular emission lines) and dust (source of the MIR continuum). At this stage, the stars are so heavily embedded that they do not appear even on the $\mathrm{K}$ maps. This locally embedding material is eventually expelled, but the clusters still reside within the kpc scale dusty star formation region, hence suffer some extinction. At this stage, the clusters appear as K extincted sources, with no MIR or radio counter parts. This interpretation is further supported by Tacconi-Garman et al. (2005): not only is the PAH-to-continuum ratio at the $\mathrm{K}$ knot positions low, but also it is high at the radio/MIR knot positions (See Fig. $4 \mathrm{~b}$ in Tacconi-Garman et al. 2005). This could mean that much of the PAH/dust around the $K$ knots is photoionised, hence weakening the $3.3 \mu \mathrm{m}$ PAH feature, has been photodissociated or has simply been blown away, while this has not yet happened in the MIR/radio knots.

\subsection{Spectra}

We have obtained NIR spectra of three embedded clusters and the nucleus: $2 \mu \mathrm{m}$ and $4 \mu \mathrm{m}$ spectra for M 6 and M 8 , and only one $2 \mu \mathrm{m}$ spectrum for M 1 and M 3. The projected slit positions are drawn in the images in Figs. 1 and 2. On these drawings, small ticks mark each arcsec, while the long and thick ticks mark the positions at which line emission has been recorded. We detect emission lines for M 1 (the nucleus), M 3, M 6 and M 8, and we even detect line emission in the region between M 6 and M 8 devoid of any conspicuous MIR or radio source. The spectra obtained for M 1, M 3, M 6 and M 8 are shown in Fig. 3.

For M 1, the continuum shown in Fig. 3 is, at the achieved angular resolution, associated to the emission line source. Three emission lines are un-ambiguously detected and measurable: $\mathrm{Pa} \alpha, \mathrm{H} 21-0 \mathrm{~S}(1)$ and $\mathrm{Br} \gamma$. The HeI $2.058 \mu \mathrm{m}$ is only tentatively detected and its flux cannot be measured because of the presence of artificial features on this part of the spectrum. Deep $2.3 \mu \mathrm{m} \mathrm{CO}$ absorption bands are detected, tracing the presence of red super giants. The equivalent width of $\operatorname{Br} \gamma$ is $4.5 \AA$. This can be compared to Starburst99 predictions (Leitherer et al. 1999), but we must keep in mind that part of the continuum, even in $\mathrm{K}$, may arise from hot dust associated to the AGN. Hence, the measured value of the equivalent width of $\mathrm{Br} \gamma$ is a lower limit. By comparing with Figs. 89 and 90 of Leitherer et al. (1999), we deduce that (1) in the instantaneous star formation case, such an equivalent width implies that the cluster is younger than $7 \mathrm{Myr}$; (2) in the continuous star formation case, there is no age constraint, since we ignore the AGN $2 \mu$ m continuum share. In conclusion, the K M 1 spectrum does not clarify the nature of the source. 

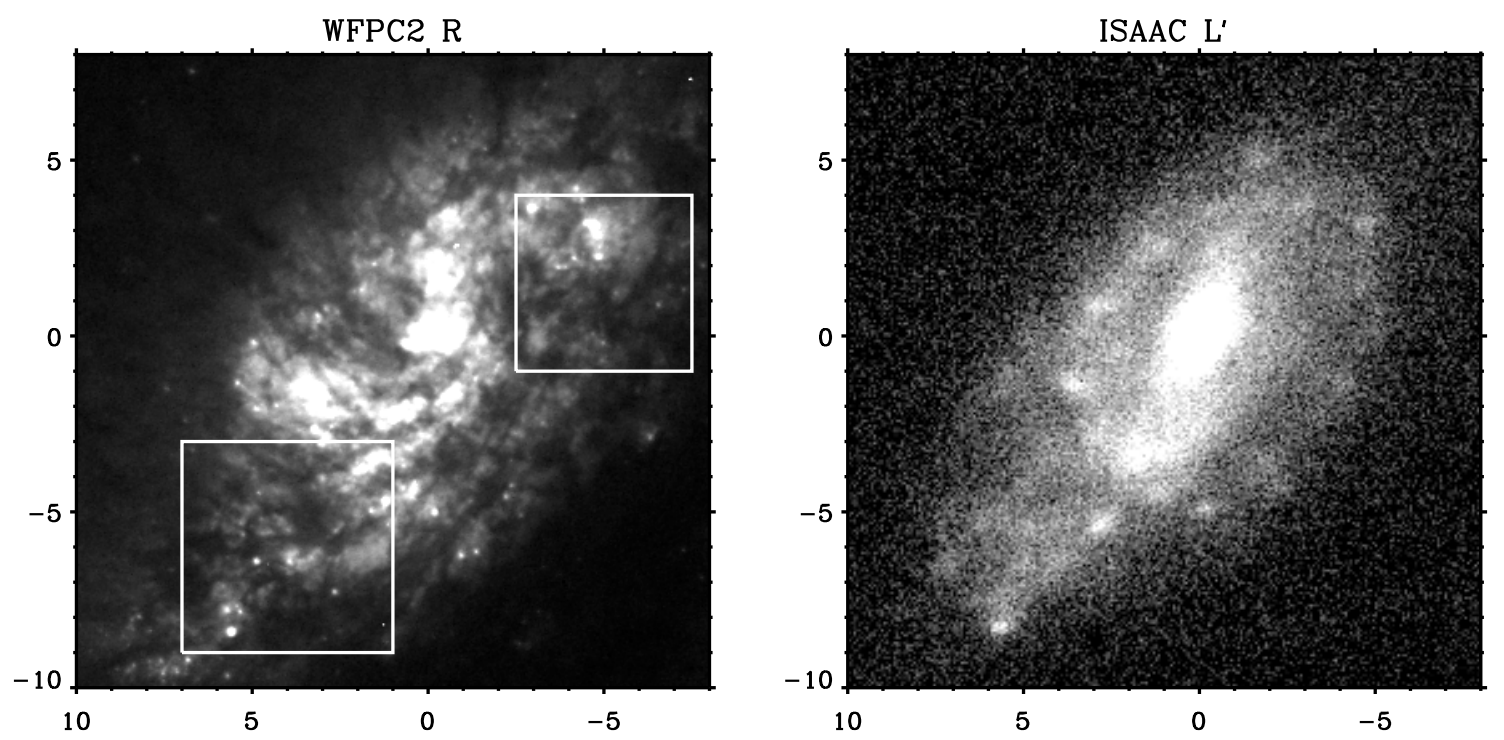

ISAAC J
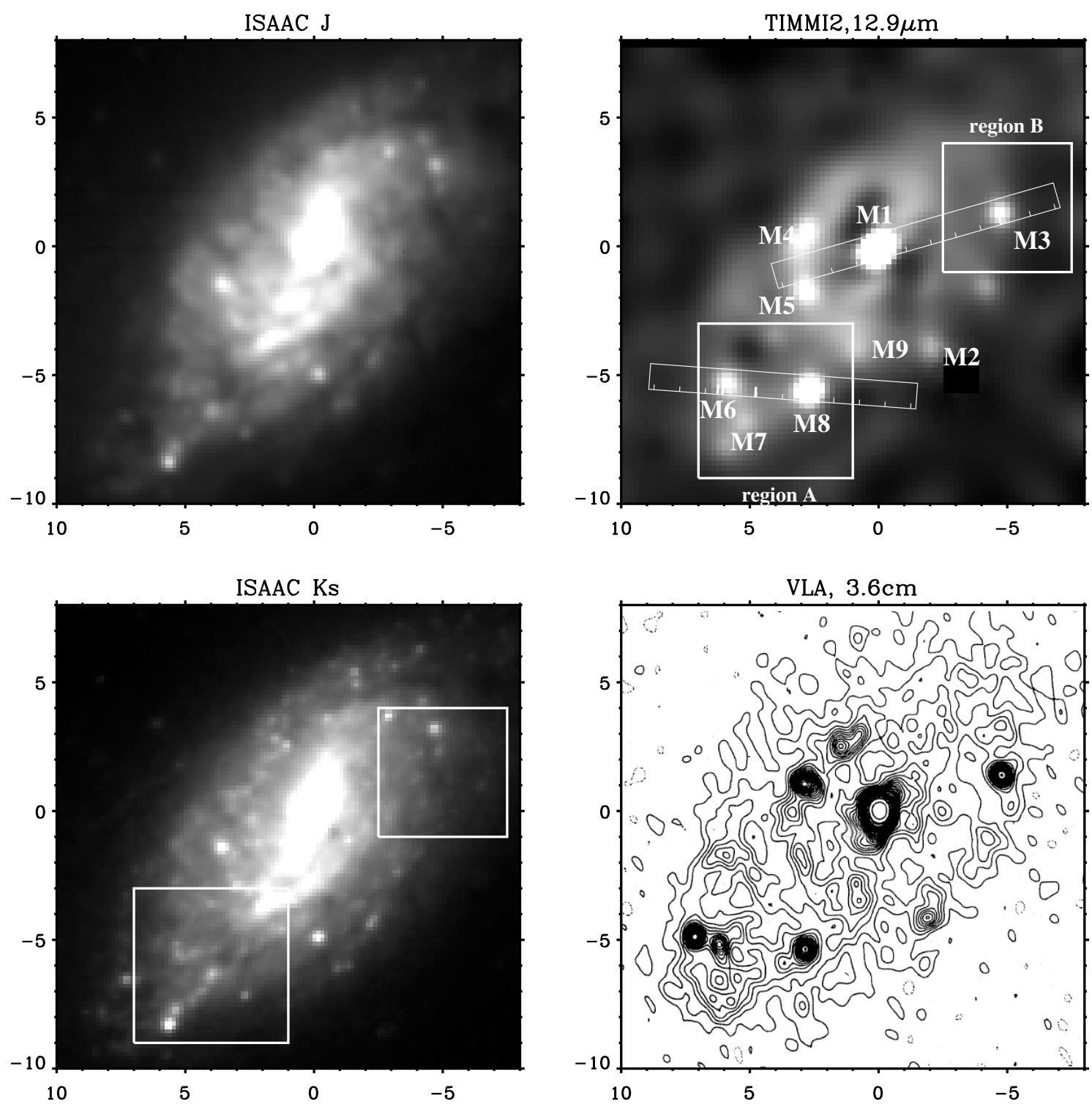

Fig. 1. The central $18^{\prime \prime} \times 18^{\prime \prime}$ region of NGC $1808\left(1^{\prime \prime}=53 \mathrm{pc}\right)$. The axis units are arcsec. The nucleus (source M 1$)$ is positioned at coordinates $\left(0^{\prime \prime} ; 0^{\prime \prime}\right)$. North is up and East is to the left. The MIR sources are labelled as in Galliano et al. (2005) on the TIMMI2 map. Zooms towards the two regions delimited by the squares (Regions A and B) are displayed in Fig. 2. The long rectangles feature the spectroscopic slits: small ticks are spaced by $1^{\prime \prime}$, while long thick ticks highlight the positions at which line emission has been recorded. 


\section{region $A$}
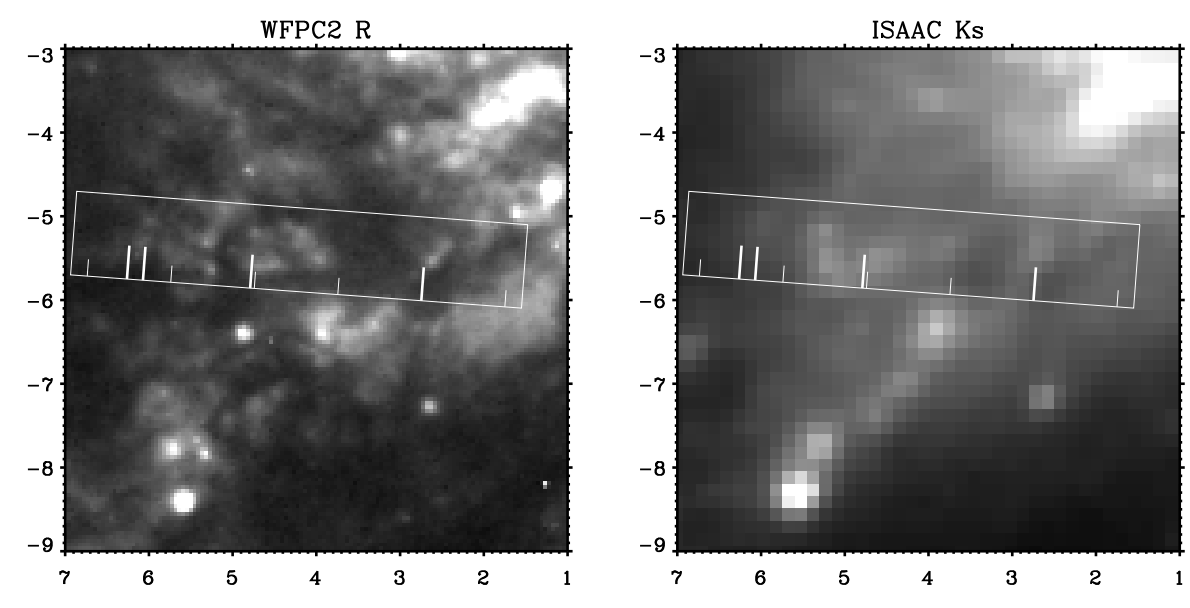

\section{region $B$}

WFPC2 R

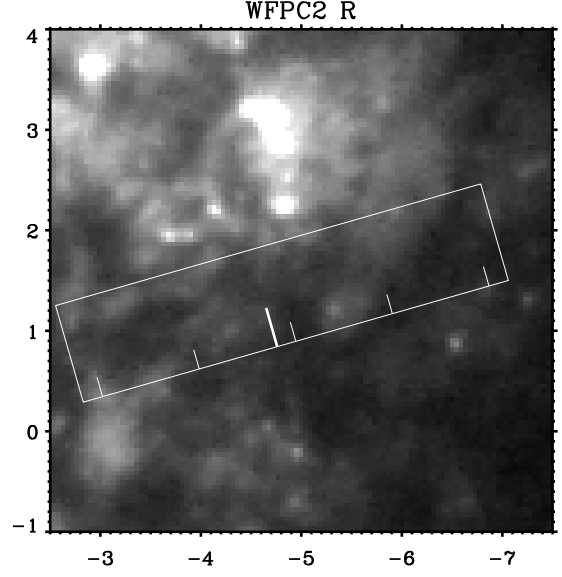

ISAAC Ks

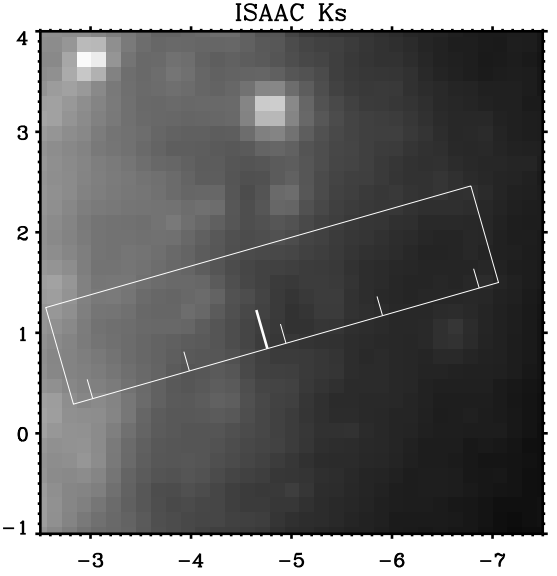

TIMMI2,12.9 $\mu \mathrm{m}$

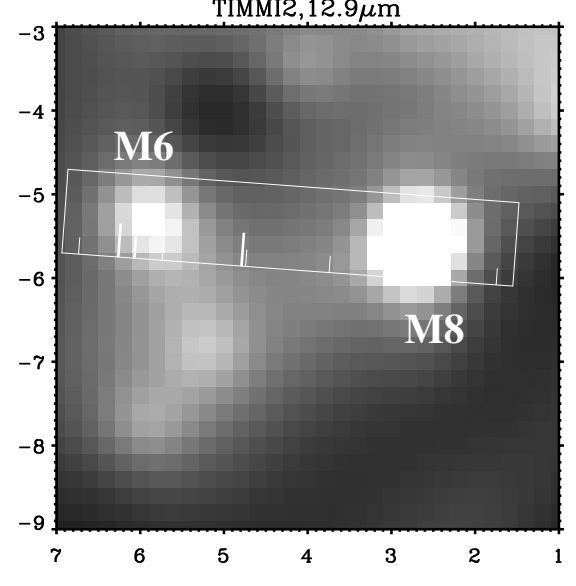

TIMMI2,12.9 $\mu \mathrm{m}$

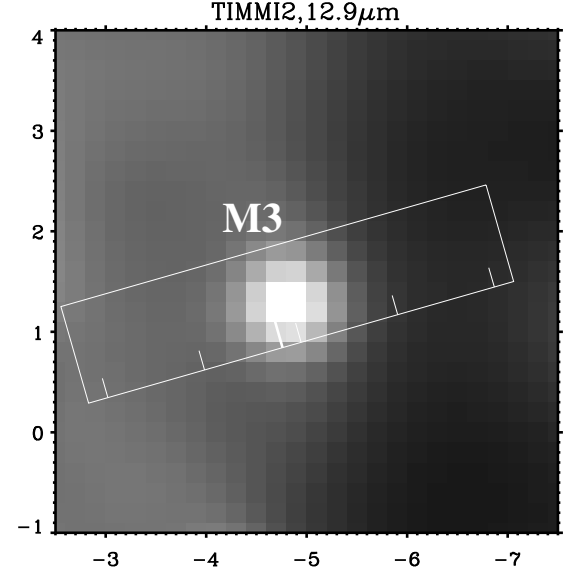

Fig. 2. Zooms towards regions A and B of the NGC 1808 central starburst. These regions are defined in Fig. 1. The axis units are arcsec, North is up and East is to the left. Regarding the slit drawings, see the caption of Fig. 1.

In the following, we focus on the three circumnuclear sources M 3, M 6 and M 8. Their spectra look quite similar in shape and are typical HII region spectra. Emission lines from atomic $(\mathrm{Pa} \alpha, \operatorname{Br} \delta$ and $\operatorname{Br} \gamma$ ) and molecular hydrogen show up. In M 6 and M 8, for which we have an additional $L^{\prime}$ spectrum, we detect the hydrogen lines $\operatorname{Pa} \gamma$ and $\operatorname{Br} \alpha$ as well as the PAH band at $3.3 \mu \mathrm{m}$. This strongly supports an interpretation of the $\mathrm{MIR} /$ radio sources in terms of heavily embedded clusters as already suggested in Galliano et al. (2005). In addition, such spectra fix an upper limit of $10 \mathrm{Myr}$ to the cluster ages, otherwise no nebular gas emission would be seen (Leitherer et al. 1999). The continuum shown on the spectra of M 3, M 6 and M 8 is not associated with the sources of emission lines. This continuum only shows the diffuse emission of the starbursting region. While the continuum emission is extended, the emission lines are only observed at the location of the MIR sources along the slit. Hence, the $\mathrm{CO}$ absorption bands seen on the spectrum continua are not associated to the embedded clusters.

Figure 4 displays a 2D spectrum for the $\operatorname{Br} \gamma$ line along the M 6-M 8 direction. The line emission extends over several $\operatorname{arcsec}(\sim 250 \mathrm{pc})$ and exhibits a complex velocity structure, suggestive of an outflow of ionised material. This probably witnesses the expelling of the locally embedding material suggested in Sect. 3.1.

\subsection{Measurements}

Table 1 provides a summary of the measurements performed on the data and gives the radio measurements of Collison et al. (1994). The following procedure has been followed for the flux density measurements and the uncertainty estimates. The relative positions of the three sources with respect to $\mathrm{M} 1$ are taken as those in the TIMMI2 $12.9 \mu \mathrm{m}$ image. They are given in the table. At these positions, we measure the flux densities inside a $0.6^{\prime \prime}$ radius aperture. The background is estimated by computing the median within an annulus with radii $0.6^{\prime \prime}$ and $1.2^{\prime \prime}$. Since, for these data, the background is difficult to interpret, we consider two values of the flux density for each source, one with and one without background subtraction, this is what we call the measurement error. We then compute an error defined as the quadratic sum of the measurement error and the photometric calibration uncertainty. The following flux calibration uncertainties are considered: $10 \%$ for the $R, J$ and $K$ s images and $20 \%$ for the $L^{\prime}$ and MIR images. The two values presented in Table 1 correspond to the final lower and upper limits of each measurement. When no clear identification of the source in the considered aperture is possible (for example, for the data in the $R, J$ and $K \mathrm{~s}$ band), we did not consider the lower limit defined above, but only the upper limit. For the line fluxes, we consider a $20 \%$ uncertainty, dominated by the calibration. Nevertheless, for the 

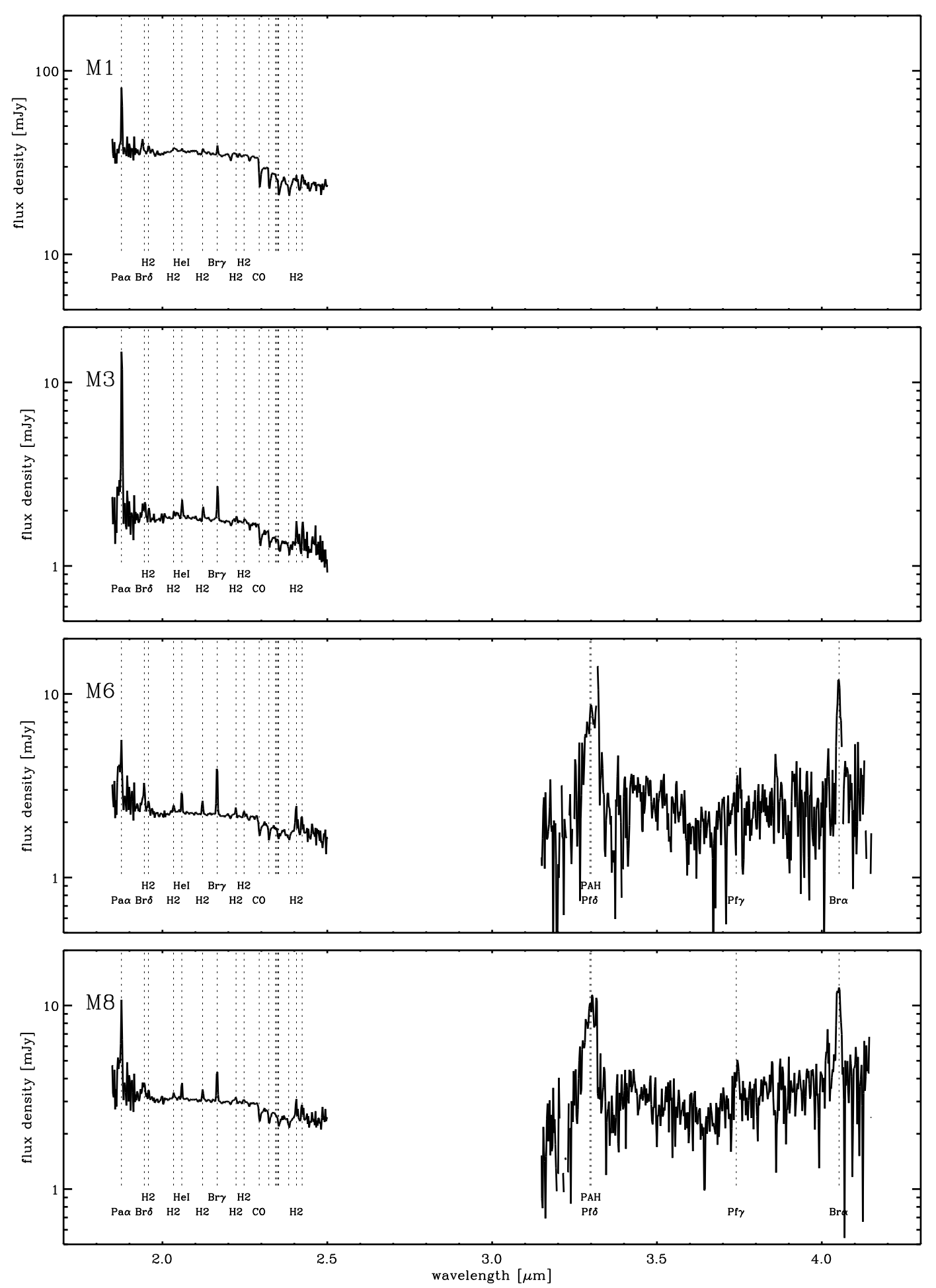

Fig. 3. ISAAC spectra of the MIR/radio sources M 1, M 3, M 6 and M 8 in the central region of NGC 1808. The slit positions, as well as the source positions along the slits are shown in Figs. 1 and 2.

lines on the blue side of the $2 \mu \mathrm{m}$ spectrum ( $\mathrm{Pa} \alpha, \mathrm{Br} \delta$ and $\mathrm{H} 21-$ $0 \mathrm{~S}(3)$ ), the uncertainty may be greater than this value because of the strong atmospheric features present in this wavelength range and the values given must be taken with caution.

\section{Cluster derived parameters}

In this section, we discuss some intrinsic parameters of the sources which can be derived from the NIR emission lines. The procedure has been applied on M 6 and M 8 , for which both the $2 \mu \mathrm{m}$ spectra (Br $\gamma$ flux) and $4 \mu \mathrm{m}$ spectra ( $\mathrm{Br} \alpha$ flux) are available. Under the assumption of simple foreground extinction, we derive the extinction towards the line emission gas, and then compute the un-extincted line luminosities. This in turn allows an estimation of the ionising photon emission rate.

We have deduced the extinction from the $\operatorname{Br} \gamma / \operatorname{Br} \alpha$ ratio. This ratio has a low dependency on temperature and density. Assuming reasonable values for the temperature and density 
Table 1. Measurements for the clusters M 1, M 3, M 6 and M 8 in NCG 1808.

\begin{tabular}{|c|c|c|c|c|c|c|c|c|}
\hline & \multicolumn{2}{|c|}{ M 1} & \multicolumn{2}{|c|}{ M3 } & \multicolumn{2}{|c|}{$\overline{\mathrm{M} 6}$} & \multicolumn{2}{|c|}{ M 8} \\
\hline & \multicolumn{6}{|c|}{ Positions with respect to M 1 [arcsec] } & \multirow{2}{*}{\multicolumn{2}{|c|}{$2.71+0.1$}} \\
\hline$\Delta \alpha[\operatorname{arcsec}]$ & \multirow{2}{*}{\multicolumn{2}{|c|}{$\begin{array}{l}0 \\
0\end{array}$}} & \multicolumn{2}{|c|}{$-1.99 \pm 0.1$} & \multicolumn{2}{|c|}{$5.82 \pm 0.1$} & & \\
\hline$\Delta \delta[\operatorname{arcsec}]$ & & & \multicolumn{2}{|c|}{$-3.76 \pm 0.1$} & \multicolumn{2}{|c|}{$-5.25 \pm 0.1$} & \\
\hline & \multicolumn{6}{|c|}{ Flux densities [mJy] } & \multicolumn{2}{|c|}{$-5.51 \pm 0.1$} \\
\hline & \multicolumn{2}{|c|}{ Value } & \multicolumn{2}{|c|}{ Value } & \multicolumn{2}{|c|}{ Value } & \multicolumn{2}{|c|}{ Value } \\
\hline & Low & High & Low & High & Low & High & Low & High \\
\hline$R$ & 2.2 & 3.1 & 0 & 0.2 & 0 & 0.2 & 0 & 0.2 \\
\hline$J$ & 11. & 20. & 0 & 1.2 & 0 & 1.3 & 0 & 1.7 \\
\hline$K \mathrm{~s}$ & 22. & 39. & 0 & 2.0 & 0 & 2.2 & 0 & 2.9 \\
\hline$L^{\prime}$ & 17. & 38. & 0 & 2.8 & 0 & 3.4 & 0.4 & 4.8 \\
\hline $10.4 \mu \mathrm{m}$ & 180. & 280. & 0 & 12. & 0 & 11. & 6.4 & 23. \\
\hline $11.9 \mu \mathrm{m}$ & 400. & 670. & 11. & 50. & 10. & 76. & 18. & 99. \\
\hline $12.9 \mu \mathrm{m}$ & 700. & 1100. & 25. & 90. & 29. & 110. & 95. & 220. \\
\hline \multicolumn{9}{|c|}{ Line fluxes $\left[10^{-15} \mathrm{erg} \mathrm{s}^{-1} \mathrm{~cm}^{-2}\right]$} \\
\hline${ }^{*} \operatorname{Pa} \alpha$ & \multicolumn{2}{|c|}{$150 . \pm 30$} & \multicolumn{2}{|c|}{$49 . \pm 10$} & \multicolumn{2}{|c|}{$9.9 \pm 2.0$} & \multicolumn{2}{|c|}{$24 . \pm 5$} \\
\hline${ }^{*} \mathrm{Br} \delta$ & \multirow{2}{*}{\multicolumn{2}{|c|}{$\begin{array}{l}<4 \\
<12 .\end{array}$}} & \multirow{2}{*}{\multicolumn{2}{|c|}{$\begin{array}{l}<1.4 \\
<1.2\end{array}$}} & \multicolumn{2}{|c|}{$3.9 \pm 0.8$} & \multirow{2}{*}{\multicolumn{2}{|c|}{$\begin{array}{c}2.8 \pm 0.6 \\
<1.6\end{array}$}} \\
\hline $\mathrm{H}_{2} 1-0 \mathrm{~S}(3)$ & & & & & & .6 & & \\
\hline $\mathrm{H}_{2} 1-0 \mathrm{~S}(2)$ & \multicolumn{2}{|c|}{$<10$. } & & & 0.8 & $=0.2$ & 1.1 & $=0.3$ \\
\hline $\mathrm{HeI}$ & & & 1.3 & $=0.3$ & 2.1 & $=0.5$ & 2.2 & $=0.5$ \\
\hline $\mathrm{H}_{2} 1-0 \mathrm{~S}(1)$ & 5.8 & $=1.2$ & 0.9 & $=0.2$ & 1.2 & $=0.3$ & 1.3 & $=0.3$ \\
\hline $\operatorname{Br} \gamma$ & 9.6 & $=1.9$ & 2.6 & $=0.6$ & 5.4 & $=1.1$ & 4.2 & $=0.9$ \\
\hline $\mathrm{H}_{2} 1-0 \mathrm{~S}(0)$ & & & & & 0.6 & $=0.2$ & 0.8 & $=0.2$ \\
\hline $\mathrm{H}_{2} 2-1 \mathrm{~S}(1)$ & & & & & 0.6 & $=0.2$ & & 6.5 \\
\hline $\mathrm{H}_{2} 1-0 \mathrm{Q}(1)$ & & & 1.0 & $=0.2$ & 1.7 & $=0.4$ & 1.5 & \pm 0.3 \\
\hline $\mathrm{H}_{2} 1-0 \mathrm{Q}(3)$ & & & 1.2 & $=0.3$ & 1.0 & $=0.2$ & 1.1 & \pm 0.3 \\
\hline $\mathrm{PAH}+\mathrm{Pf} \delta$ & & & & & 84. & $=17$. & 100. & \pm 20 . \\
\hline Pf $\gamma$ & & & & & 3.2 & $=0.7$ & 5.4 & \pm 1.1 \\
\hline $\operatorname{Br} \alpha$ & & & & & 20. & \pm 4 . & 25. & \pm 5 . \\
\hline & & Radio c & $\mathrm{a}(\mathrm{Col}$ & ison & $.1994)$ & & & \\
\hline$\alpha_{3.6 \mathrm{~cm}}^{6 \mathrm{~cm}}$ & & & & & & & & .57 \\
\hline $3.6 \mathrm{~cm}[\mathrm{mJy}]$ & & & & & & & & 76 \\
\hline
\end{tabular}

* These flux values and their associated uncertainties must be taken with caution since the atmospheric features are strong in this region of the spectrum.

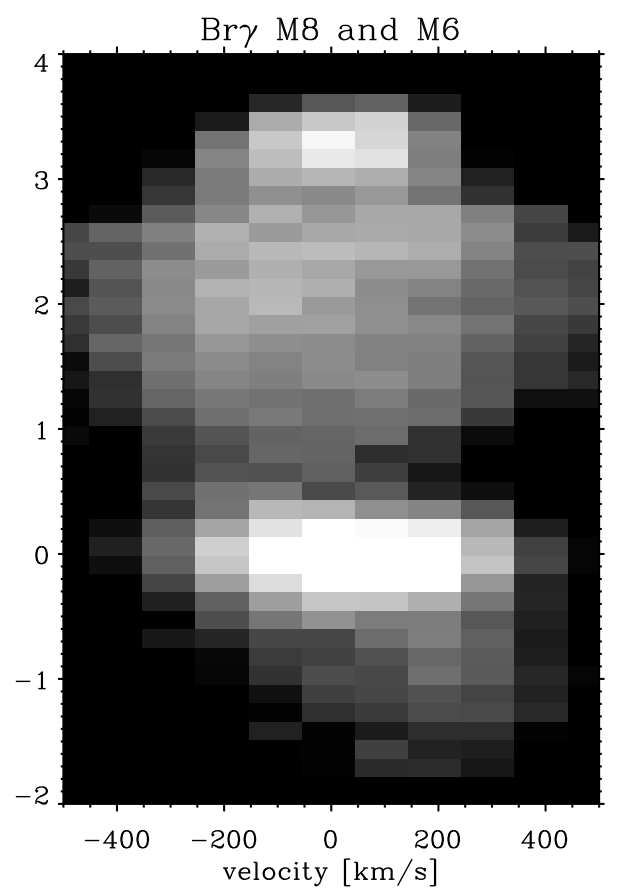

Fig. 4. Position-velocity diagram for $\mathrm{Br} \gamma$ along the slit which contains the two embedded clusters M 6 and M 8. The $X$-axis shows the wavelength expressed in relative radial velocity in $\mathrm{km} \mathrm{s}^{-1}$ and the $Y$-axis gives the relative angular position in arcsec. of HII regions, $T_{\mathrm{e}}=10^{4} \mathrm{~K}$ and $\mathrm{N}_{\mathrm{e}}=10^{4} \mathrm{~cm}^{-3}$ (Osterbrock 1989), we obtain figures of $A_{\mathrm{v}}=10.2$ and 15.0 , for $\mathrm{M} 6$ and $\mathrm{M} 8$ respectively. This leads to un-extincted $\mathrm{Br} \gamma$ fluxes of $8.9 \times 10^{-15} \mathrm{erg} \mathrm{s}^{-1} \mathrm{~cm}^{-2}$ in $\mathrm{M} 6$, and $9.5 \times 10^{-15} \mathrm{erg} \mathrm{s}^{-1} \mathrm{~cm}^{-2}$ in M 8.

For an embedded young star cluster, the ionisation-bounded case is very likely: the HII region is optically thick in the Lyman continuum and all the stellar ionising photons are absorbed. Then, the number of hydrogen ionising photons $(\lambda \leq 912 \AA)$ is directly proportional to the flux in any specific recombination line.

From Osterbrock (1989),

$Q\left[\mathrm{H}^{+}\right]=\frac{\alpha_{B}}{\alpha_{H \alpha}^{\mathrm{eff}}} \times \frac{L_{\mathrm{H} \alpha}}{h v_{\mathrm{H} \alpha}}$, where $\alpha_{B} / \alpha_{H \alpha}^{\mathrm{eff}} \sim 2.96$.

In case $\mathrm{B}$, we get $L_{\mathrm{H} \alpha} / L_{\mathrm{B} \gamma}=103.6$. The ionising photon emission rates of the two embedded clusters can then be derived and are found to be around $9.6 \times 10^{51} \mathrm{~s}^{-1}$ and $10.2 \times 10^{51} \mathrm{~s}^{-1}$, in M 6 and M 8 respectively. This corresponds to about 1000 O6 stars in each cluster.

The way we have treated extinction in the above computation is a crude one. This is undoubtly a first order estimate which calls for a future, more realistic approach, once additional data constraints will become available. Yet, the interpretation of the MIR/radio sources in terms of embedded young clusters is confirmed and, in addition, an upper limit on the cluster ages is obtained at $10 \mathrm{Myr}$. 


\section{Conclusions}

The main conclusions are:

- None of the MIR/radio sources unveiled by Galliano et al. (2005) can be detected in the $J, K$ s and even $L^{\prime}$ band images, except for M 8 in the $L^{\prime}$ band.

- For three of the sources, namely M 3, M 6 and M 8, we have collected NIR spectra: they display intense nebular emission lines, confirming that the sources are young embedded clusters.

- The fact that the MIR/radio sources do not show up in the NIR images demonstrates that such embedded clusters must be searched for primarily in the MIR or at radio wavelengths.

- A complex velocity structure in the ionised gas is observed around sources M 6 and M 8, suggesting the presence of cluster winds.

- For two sources, M 6 and M 8, the extinction towards the nebular gas has been estimated, with values of $A_{\mathrm{v}}=10.2$ in M 6 and $A_{\mathrm{v}}=15$ in M 8.

- The extinction-corrected Br $\gamma$ fluxes for M 6 and M 8 imply ionising photon emission rates of respectively $9.6 \times 10^{51} \mathrm{~s}^{-1}$ and $10.2 \times 10^{51} \mathrm{~s}^{-1}$.

A more complete analysis of the deeply embedded cluster parameters, in particular the derivation of their age and mass, requires some knowledge of their MIR spectral features (Galliano et al. 2008) and it is left for a subsequent analysis, after spectral MIR data will have been collected.

Acknowledgements. We thank the daytime and nighttime support staff at Cerro Paranal Observatory, who made these observations possible, and the anonymous referee for the quick reply. E.G. thanks the ESO fellowship program and the PCI program of ON/MCT (DTI/CNPq grant number 383076/07-2).

\section{References}

Collison, P. M., Saikia, D. J., Pedlar, A., Axon, D. J., \& Unger, S. W. 1994, MNRAS, 268, 203

Galliano, E., Alloin, D., Pantin, E., Lagage, P. O., \& Marco, O. 2005, A\&A, 438, 803

Galliano, E., Alloin, D., Pantin, E., et al. 2008, A\&A, submitted

Jiménez-Bailón, E., Santos-Lleó, M., Dahlem, M., et al. 2005, A\&A, 442, 861

Koribalski, B., Dettmar, R.-J., Mebold, U., \& Wielebinski, R. 1996, A\&A, 315, 71

Koribalski, B. S., Staveley-Smith, L., Kilborn, V. A., et al. 2004, AJ, 128, 16

Krabbe, A., Böker, T., \& Maiolino, R. 2001, ApJ, 557, 626

Leitherer, C., Schaerer, D., Goldader, J. D., et al. 1999, ApJS, 123, 3

Osterbrock, D. E. 1989, Astrophysics of gaseous nebulae and active galactic nuclei, Research supported by the University of California, John Simon Guggenheim Memorial Foundation, University of Minnesota (Mill Valley, CA: University Science Books), 422

Tacconi-Garman, L. E., Sternberg, A., \& Eckart, A. 1996, AJ, 112, 918

Tacconi-Garman, L. E., Sturm, E., Lehnert, M., et al. 2005, A\&A, 432, 91 